J. Lake Sci.(湖泊科学), 2020, 32(3): 887-898

DOI 10. 18307/2020. 0327

(c) 2020 by Journal of Lake Sciences

\title{
蓝藻不同类型胶被提取方法
}

\author{
刘冷昕 ${ }^{1,2}$, 肖 艳 ${ }^{1 * *}$, 李 哲 ${ }^{1}$, 吴兴华 ${ }^{3}$, 张 宇 $^{3}$ \\ (1: 中国科学院重庆绿色智能技术研究院,中国科学院水库水环境重点实验室,重庆 400714) \\ (2: 中国科学院大学, 北京 100049) \\ (3: 中国长江三峡集团有限公司, 北京 100038)
}

\begin{abstract}
摘 要: 胶被作为蓝藻细胞外的一层功能性结构, 其提取方法仍不清晰完善. 本研究选取具有不同胶被形态的鱼腥藻 $A n-$ abaena sp. 和念珠藻 Nostoc sp. 为实验材料, 通过归纳近 10 年有关藻类胶被的提取方法, 总结影响胶被提取效果的主要因 素(温度、提取剂浓度、时间等) 范围,并设置一系列因素及其梯度的双因素实验和正交实验对胶被提取方法进行优化, 观 察提取过程中藻细胞形态、自发荧光以及藻细胞密度变化. 结果表明, 粘液可通过离心法与藻细胞分离; 英膜提取的硫酸 水浴法的最优条件为: $1 \mathrm{~mol} / \mathrm{L} 、 60^{\circ} \mathrm{C} 、 30 \mathrm{~min}$, 氢氧化钠水浴法为: $0.01 \mathrm{~mol} / \mathrm{L} 、 50^{\circ} \mathrm{C} 、 20 \mathrm{~min}$; 鞘提取的最优方法条件为氢氧

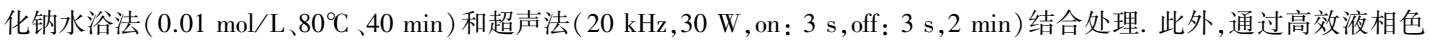
谱法测定提取的蓝藻胶被多糖组分, 结果显示, 鞘具有较强的疏水性, 未水化为粘液或 RPS, 而荚膜中含有的氨基葡萄糖 $(1.261 \pm 0.02 \mathrm{mg} / \mathrm{g})$ 使得英膜比鞘更具有粘附力. 将本研究得到的英膜和鞘的最优提取法运用到胶被形态以英膜和鞘为 主的其他藻种,均有很好的提取效果. 本研究可为后续进一步揭示不同类型胶被的功能作用提供技术支撑.
\end{abstract}

关键词: 蓝藻; 胶被提取;形态;方法优化;氨基葡萄糖

\section{Extraction methods of different morphotypes of surface coat in cyanobacteria"}

\author{
LIU Lingxin ${ }^{1,2}$, XIAO Yan $^{1 * *}$, LI Zhe ${ }^{1}$, WU Xinghua ${ }^{3} \&$ ZHANG Yu ${ }^{3}$ \\ ( 1: Key Laboratory of Reservoir Aquatic Environment, Chongqing Institute of Green and Intelligent Technology, Chinese Acad- \\ emy of Sciences, Chongqing 400714, P.R.China) \\ (2: University of Chinese Academy of Sciences, Beijing 100049, P.R.China) \\ (3: China Three Gorges Corporation, Beijing 100038, P.R.China)
}

\begin{abstract}
Surface coat is a functional structure surrounding cells of cyanobacteria, and the extraction method is still not well compared and consistently reported. Previous work suggested that the main factors affecting the extraction of cyanobacterial surface coat were temperature, extractant concentration, extraction time, etc. To develop the optimal extraction methods for surface coat of cyanobacteria, Anabaena sp. and Nostoc sp. with different morphotypes of surface coat were selected. We performed a series of factors based on two-factor and orthogonal experimental design. Through comparison of cell morphology, spontaneous fluorescence and cell density after surface coat extraction, the results showed that slime can be separated from cyanobacterial cells by centrifugal method. Both sulfuric acid and sodium hydroxide water-bath methods can extract capsule, and the optimal conditions were $1 \mathrm{~mol} / \mathrm{L}, 60^{\circ} \mathrm{C}$, $30 \mathrm{~min}$ for sulfuric acid extraction and $0.01 \mathrm{~mol} / \mathrm{L}, 50^{\circ} \mathrm{C}, 20 \mathrm{~min}$ for sodium hydroxide, respectively. The optimal method of sheath extraction was $0.01 \mathrm{~mol} / \mathrm{L}$ sodium hydroxide water-bath method, $80^{\circ} \mathrm{C}, 40 \mathrm{~min}$ and $20 \mathrm{kHz}, 30 \mathrm{~W}$, on: $3 \mathrm{~s}$, off: $3 \mathrm{~s}, 2$ min by ultrasonic method. In addition, the polysaccharide components of extracted surface coat examination by high-performance liquid chromatography (HPLC) indicated that the sheath is highly hydrophobic and is not hydrated to slime or RPS in the culture medium. The glucosamine $(1.261 \pm 0.02 \mathrm{mg} / \mathrm{g})$ contained in capsule makes the capsule more adhesion than the sheath. Additionally, the optimal extraction methods obtained from this study can be effectively applied to other cyanobacterial and green algal species whose surface coats are mainly capsule and sheath. This study may provide technical support for further revealing the biological
\end{abstract}

* 2019-09-03 收稿; 2019-10-22 收修改稿.

国家自然科学基金项目 (51779240, 51979262)资助.

** 通信作者;E-mail:yxiao@ cigit.ac.cn. 
function of cyanobacterial and algal surface coat.

Keywords: Cyanobacteria; surface coat extraction; morphotype; method optimization; glucosamine

胶被 ( surface coat) 是一种广泛存在于微生物以及一些植物和动物细胞周围的结构. 由于其主要成分为 多糖和蛋白质, 早期的学者提出一个广义的术语“ glycocalyx ” 来描述这种功能性结构 ${ }^{[1]}$. 在过去的几十年 里, 微生物和植物的胶被得到了越来越多的关注, 但其术语经常混淆, 特别是藻类的胶被, 在许多文献中其 名称并没有严格区分. 在已有的研究报道中, 藻类的胶被称为 capsular polysaccharides (CPS) 、bound extracellular polymers(BEPS)、 tight extracellular polymers (TEPS) 、transparent exopolymer particles ( TEP) 、bound extracellular organic matter(bEOM)、polysaccharide (PS) 等 ${ }^{[2-4]}$, BEPS 还可进一步分为 loosely bound extracellular polymers (LB-EPS) 和 tightly bound extracellular polymers (TB-EPS $)^{[5]}$. 除此之外, 大部分藻类胶被可以分泌溶 解性多糖物质到外界环境中, 一般称之为 released polysaccharides (RPS) 或 soluble extracellular polymers (SEPS), 因此有学者用胞外多糖 (exopolysaccharide、extracellular polymeric substance、EPS) 代表胶被及其分泌 物, 在物质层面上对其进行分析 ${ }^{[6-7]}$. 但这忽略了藻类胶被自身的生物学功能, 且胶被可能比 RPS 具有更多 的生物活性 ${ }^{[8]}$.

已有的研究表明,胶被的形成和保留可作为藻类与其周围环境之间的物理屏障, 同时也为细胞周围提 供了必要营养素和微量元素的微环境 ${ }^{[5,9]}$. 尤其是蓝藻和绿藻的胶被, 被认为对紫外线辐射、氧化、有毒化合 物、干燥和原生动物的捕食等不利环境因素具有防御作用 ${ }^{[10-12]}$. 此外, 这些外层对于蓝藻和绿藻维持其群体 形态至关重要, 并参与细胞间相互作用、粘附和聚集等一系列过程, 从而进一步影响蓝藻和绿藻水华的形 成 ${ }^{[13-14]}$. 然而, 许多学者对藻类胶被并没有系统深人地区别划分, 导致这些外层之间的结构和功能研究仍然 是零散和片面的.

研究者普遍认为, 藻类胶被根据形态可分为鞘 ( sheath)、荚膜 ( capsule)、粘液 ( slime $)^{[15]}$. 鞘层薄而均 匀, 在光学显微镜下观察可见; 英膜是厚而有粘性的多糖层, 用印度墨水染色后光学显微镜下观察可见; 粘 液是最外一层, 分散在细胞周围, 不能反映细胞的形状 ${ }^{[15]}$. 而目前有关藻类不同类型胶被的研究相对较少, 且针对不同类型的胶被如何在保证细胞完整状态下被完全提取的方法仍不清晰. 归纳近 10 年研究中关于 藻类胶被的提取方法 (表 1), 目前最常用的方法有离心法、热水提取法、氢氧化钠水浴法等. 然而不同藻种 的胶被提取方法不一, 即使是同一藻种, 如铜绿微囊藻 (Microcystis aeruginosa), 不同的文献所提及的方法以 及采用的提取时间、提取剂浓度、温度也均有不同 ${ }^{[5,16-19]}$, 且大部分文献中并未对特定的藻种胶被形态进行 描述. 现有文献提取胶被采用温度范围广, 研究表明高温会促进多糖的溶解 ${ }^{[20]}$, 最高达到 $100^{\circ} \mathrm{C}$; 提取时间 范围为 $10 \mathrm{~min}$ 到 $24 \mathrm{~h}$,Frølund 等发现 $0.5 \sim 1 \mathrm{~h}$ 提取胶被对导致细胞裂解的风险最小 ${ }^{[21]}$, Fang 等也发现胶被 提取完成时间少于 $1 \mathrm{~h}$ 最佳 ${ }^{[22]}$.

藻类胶被提取方法的不完善, 以及在保证细胞完整的条件下针对不同类型胶被的提取方法缺失,很大 程度上阻碍了对藻类胶被生物学功能的深人探索. 此外, 不同藻种胶被形态、性质不同, 其提取方法也不尽 相同, 胶被提取效果不完全也为后续分析带来一定误差, 因此首先寻找适宜的胶被提取方法十分重要. 基于 此背景, 本研究选取水华优势种鱼腥藻 Anabaena sp. 和念珠藻 Nostoc sp. 为研究对象, 探讨其胶被类型以及相 对应的最优提取方法,为后续进一步揭示不同类型胶被的功能作用提供技术支撑.

\section{1 材料与方法}

\section{1 藻种及藻种培养}

实验选用的鱼腥藻 FACHB-82 Anabaena sp. (具副异形胞, 藻丝所有细胞形态相同) 和念珠藻 FACHB599 Nostoc sp. (具离异形胞, 藻体单生, 成熟后为球形), 由中国科学院水生生物研究所淡水藻种库 (FACHBCollection) 提供, 藻种常规培养以 BG-11 为基础培养基, 培养温度为 $25 \pm 1^{\circ} \mathrm{C}$, 光照强度为 $25 \mu$ mol photons/ $\left(\mathrm{m}^{2} \cdot \mathrm{s}\right)$, 光周期为 $12 \mathrm{~h}: 12 \mathrm{~h}$, 每天手动摇匀 3 次.

\section{2 藻种胶被形态观察}

在倒置苂光显微镜( OLYMPUS IX73) 明场下观察藻类胶被的鞘结构,通过印度墨水(PH1714, phygene) 
表 1 近 10 年有关藻类胶被提取方法归纳

Tab.1 Summary of extraction methods of algal surface coat in recent ten years

\begin{tabular}{|c|c|c|c|c|}
\hline 提取方法 & 藻种( 拉丁名) & 胶被 & 实验条件 & 参考文献 \\
\hline \multirow[t]{4}{*}{ 离心法 } & 铜绿微囊藻 (Microcystis aeruginosa) & EPS & $25^{\circ} \mathrm{C}, 10000 g, 10 \mathrm{~min}$ & {$[16]$} \\
\hline & 小球藻 ( Chlorella sp. ) & B-EPS & $4^{\circ} \mathrm{C}, 10000 g, 15 \min$ & {$[23]$} \\
\hline & 盐生杜氏藻 (Dunaliella salina) & EPSs & $4^{\circ} \mathrm{C}, 15000 g, 20 \mathrm{~min}$ & {$[24]$} \\
\hline & 铜绿微囊藻 (Microcystis aeruginosa) & EPS & $25^{\circ} \mathrm{C}, 14000 \mathrm{r} / \mathrm{min}, 20 \mathrm{~min}$ & {$[17]$} \\
\hline \multirow[t]{4}{*}{ 热水提取法 } & 铜绿微囊藻 (Microcystis aeruginosa) & TB-EPS & $0.05 \% \mathrm{NaCl}, 60^{\circ} \mathrm{C}, 30 \mathrm{~min}$ & {$[5,25]$} \\
\hline & 念珠藻 ( Nostoc sp. ) & capsule/slime & 蒸馏水, $80^{\circ} \mathrm{C}, 6 \mathrm{~h}$ & {$[26-27]$} \\
\hline & 微鞘藻 (Microcoleus sp. ) & CPS & 蒸馏水, $80^{\circ} \mathrm{C}, 6 \mathrm{~h}$ & {$[8]$} \\
\hline & 蓝螺藻( Cyanobacteria) & CPS & $1.5 \% \mathrm{NaCl}, 80^{\circ} \mathrm{C}, 4 \mathrm{~h}$ & {$[7]$} \\
\hline \multirow[t]{4}{*}{ 氢氧化钠水浴法 } & 铜绿微囊藻 (Microcystis aeruginosa) & BEPS & $\mathrm{NaOH}(\mathrm{pH}=10), 45^{\circ} \mathrm{C}, 4 \mathrm{~h}$ & {$[18]$} \\
\hline & 惠氏微囊藻 (Microcystis wesenbergii) & bEPS & $\mathrm{NaOH}(\mathrm{pH}=10), 45^{\circ} \mathrm{C}, 4 \mathrm{~h}$ & {$[28]$} \\
\hline & 斜生栅藻( Scenedesmus obliquus) & TB-EPS & $0.5 \mathrm{~mol} / \mathrm{L} \mathrm{NaOH}, 100^{\circ} \mathrm{C}, 15 \mathrm{~min}$ & {$[19]$} \\
\hline & 铜绿微囊藻( Microcystis aeruginosa) & TB-EPS & $0.5 \mathrm{~mol} / \mathrm{L} \mathrm{NaOH}, 100^{\circ} \mathrm{C}, 15 \mathrm{~min}$ & {$[19]$} \\
\hline 盐酸水浴法 & 指状梭形鼓藻( Netrium digitus) & mucilage & $0.1 \mathrm{~mol} / \mathrm{L} \mathrm{HCl}, 25^{\circ} \mathrm{C}, 15 \sim 40 \mathrm{~min}$ & {$[29]$} \\
\hline EDTA 提取法 & 螺旋藻 ( Spirulina sp. ) & TEPS & $0.1 \mathrm{~mol} / \mathrm{L} \mathrm{EDTA}, 25^{\circ} \mathrm{C}, 4 \mathrm{~h}$ & {$[6]$} \\
\hline \multirow[t]{2}{*}{ 阳离子交换树脂法 } & 舟形藻 ( Navicula sp. ) & bEPS & 强酸型阳离子交换树脂, $\mathrm{PBS}, 4^{\circ} \mathrm{C}, 1 \mathrm{~h}$ & {$[30]$} \\
\hline & 硅藻( Diatom) & bEPS & 强酸型阳离子交换树脂, $\mathrm{PBS}, 4^{\circ} \mathrm{C}, 1 \mathrm{~h}$ & {$[31]$} \\
\hline 蛋白酶法 & 螺旋藻 ( Spirulina sp. ) & EPS & 碱性蛋白酶, $50^{\circ} \mathrm{C}, 24 \mathrm{~h}$ & {$[3]$} \\
\hline
\end{tabular}

染色观察藻细胞胶被的荚膜和粘液, 分别对藻种进行胶被形态的分类.

本研究所选藻种鱼腥藻呈群体形态, 许多藻丝聚集在一起 (图 1a), 但群体和单根藻丝外没有鞘层 (图 $1 \mathrm{~b}$ ); 印度墨水染色发现鱼腥藻群体中存在大量荚膜和粘液, 包裹在藻丝周围(图 1c). 念珠藻群体中许多藻 丝聚集在一起, 在未染色情况下观察到群体外存在较厚的鞘层结构 (图 1d); 印度墨水染色下, 念珠藻群体 鞘外未存在其他形态的胶被 (图 1e).
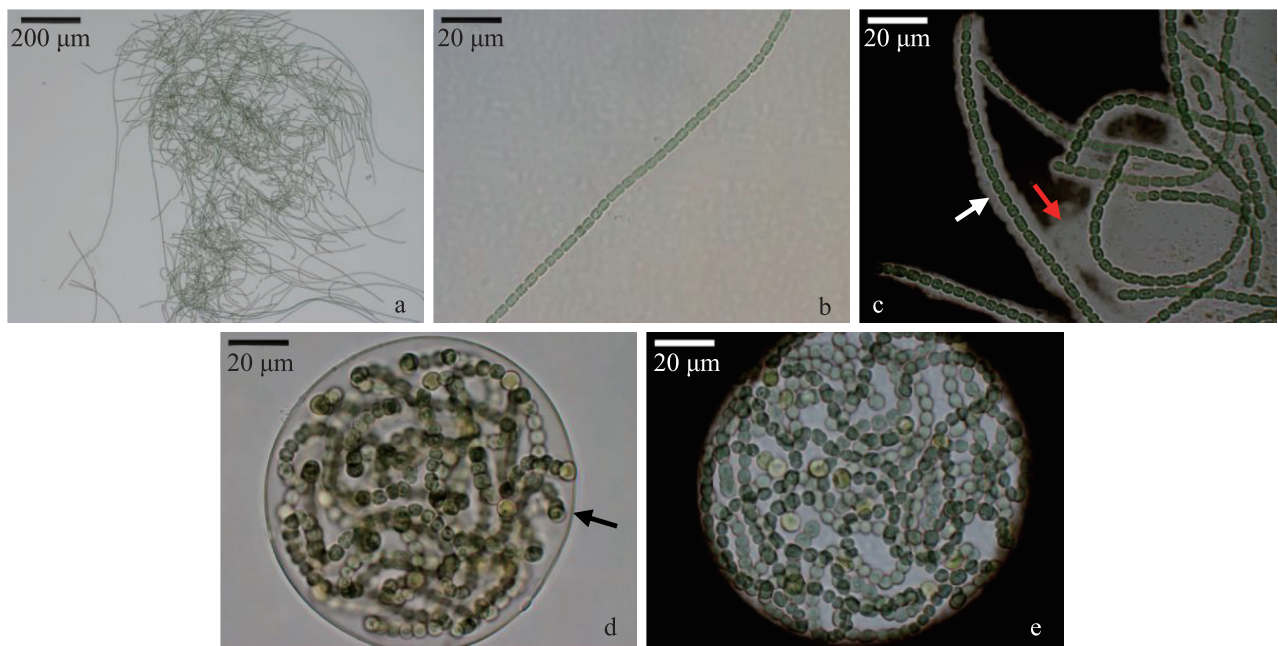

图 1 鱼腥藻和念珠藻的胶被形态: ( a c) FACHB-82 鱼腥藻; ( d e)FACHB-599 念珠藻 ( $\mathrm{a} 、 \mathrm{~b}$ : 未染色; $\mathrm{c}$ : 印度墨水染色, 荚膜(白箭头), 粘液 (红箭头) ; d : 未染色,鞘 (黑箭头); $\mathrm{e}$ :印度墨水染色)

Fig.1 Surface coat of Anabaena and Nostoc: (a-c) FACHB-82 Anabaena sp.; (d-e) FACHB-599 Nostoc sp.

( a, b: unstained; c: stained with India ink, capsule (white arrow), slime ( red arrow); d: unstained, sheath (black arrow); e: stained with India ink) 


\section{3 实验设计}

取对数生长中期藻液 $30 \mathrm{~mL}$, 用 $0.22 \mu \mathrm{m}$ 醋酸纤维滤膜过滤, 滤液即为 RPS, 后用透析袋 (1000-Da) 在去 离子水中纯化 RPS. 将滤膜表面的藻样重悬于 $30 \mathrm{~mL}$ 的 $\mathrm{NaCl}$ 溶液 ( $0.05 \%$, 维持藻细胞渗透压) 中, 以 $10000 \mathrm{~g}, 4^{\circ} \mathrm{C}$ 下离心 $10 \mathrm{~min}$, 收集上清液即为粘液, 后用 $0.22 \mu \mathrm{m}$ 醋酸纤维滤膜过滤.

根据表 1 中归纳的胶被提取方法的普适范围, 设置一系列温度、时间、提取剂浓度梯度,将离心沉淀和 滤膜表面的藻细胞处理如下: (1)用 $\mathrm{NaCl}$ 溶液 (0.05\%) 重悬浮到 $30 \mathrm{~mL}$, 进行双因素实验处理 (表 2); (2)用 $\mathrm{H}_{2} \mathrm{SO}_{4} 、 \mathrm{NaOH}$ 提取剂重悬浮到 $30 \mathrm{~mL}$, 进行正交实验处理(表 3), 每 $10 \mathrm{~min}$ 震荡 1 次. 由于念珠藻鞘层较坚 硬,因此在正交实验中温度设计较高.

表 2 双因素实验设计

Tab.2 Two-factor experimental design

\begin{tabular}{|c|c|c|c|c|c|c|c|}
\hline \multicolumn{2}{|c|}{ 离心法 } & \multicolumn{2}{|c|}{ 冰浴超声法 } & \multicolumn{2}{|c|}{ 热水提取法 } & \multicolumn{2}{|c|}{ 阳离子交换树脂法 } \\
\hline 离心速度 & 时间/min & 超声功率/W & 时间/min & 温度 $/{ }^{\circ} \mathrm{C}$ & 时间/min & 树脂量/g & 时间/min \\
\hline $15000 g$ & 10 & 30 & 1 & 50 & 10 & 1 & 20 \\
\hline $20000 g$ & 20 & 60 & 2 & 60 & 20 & 2 & 40 \\
\hline $25000 g$ & 30 & 90 & 3 & 70 & 30 & 3 & 60 \\
\hline $30000 \mathrm{~g}$ & 40 & 120 & 4 & 80 & 40 & 4 & 80 \\
\hline
\end{tabular}

表 3 正交实验设计

Tab.3 Orthogonal experimental design

\begin{tabular}{|c|c|c|c|c|c|c|}
\hline \multirow{3}{*}{ 实验编号 } & \multicolumn{6}{|c|}{ 硫酸水浴法/氢氧化钠水浴法 } \\
\hline & \multicolumn{3}{|c|}{ 鱼腥藻 } & \multicolumn{3}{|c|}{ 念珠藻 } \\
\hline & 提取剂 ${ }^{1}$ 浓度/( mol/L) & 温度 $/{ }^{\circ} \mathrm{C}$ & 时间 $/ \mathrm{min}$ & 提取剂 ${ }^{1)}$ 浓度/( mol/L) & 温度 $/{ }^{\circ} \mathrm{C}$ & 时间 $/ \mathrm{min}$ \\
\hline 1 & 0.01 & 50 & 20 & 0.01 & 60 & 20 \\
\hline 2 & 0.01 & 60 & 40 & 0.01 & 70 & 30 \\
\hline 3 & 0.01 & 70 & 30 & 0.01 & 80 & 40 \\
\hline 4 & 0.1 & 50 & 30 & 0.1 & 60 & 30 \\
\hline 5 & 0.1 & 60 & 20 & 0.1 & 70 & 40 \\
\hline 6 & 0.1 & 70 & 40 & 0.1 & 80 & 20 \\
\hline 7 & 1 & 50 & 40 & 1 & 60 & 40 \\
\hline 8 & 1 & 60 & 30 & 1 & 70 & 20 \\
\hline 9 & 1 & 70 & 20 & 1 & 80 & 30 \\
\hline
\end{tabular}

1) 硫酸水浴法提取剂: $\mathrm{H}_{2} \mathrm{SO}_{4}$; 氢氧化钠水浴法提取剂: $\mathrm{NaOH}$.

上述处理后的样品在 $4^{\circ} \mathrm{C}$ 下以 $5000 \mathrm{~g}$ 离心 $10 \mathrm{~min}$, 收集上清液即为荚膜/鞘. 将离心沉淀的藻细胞重悬 于 $\operatorname{NaCl}(0.05 \%)$ 中, 通过印度墨水染色观察藻细胞胶被是否提取完全. 对于胶被均能完全提取的处理组, 于 苂光显微镜 ( OLYMPUS IX73) G 模式下观察藻细胞自发苂光, 激发波长为 $530 \sim 550 \mathrm{~nm}$, 测定藻细胞数, 确定 胶被最优提取方法.

\section{4 测定指标及方法}

1.4.1 生物量及细胞数的测定 取对数生长中期藻液 $30 \mathrm{~mL}$ 用于测定生物量及细胞密度. 生物量以藻细胞干 重 $\left(\mathrm{DW}\right.$ ) 表示, 将藻液用 GF/F 滤膜( Whatman, UK) 抽滤, 滤膜提前烘干至恒重并记录重量 $W_{0}(\mathrm{~g})$, 后将滤有 藻样的滤膜置于 $60^{\circ} \mathrm{C}, 24 \mathrm{~h}$ 烘干至恒重, 记录重量 $W_{t}(\mathrm{~g})$. 生物量 $X=1000 \times\left(W_{t}-W_{0}\right) / V$, 其中, $V$ 为藻液体 积 $(\mathrm{mL})$.

采用血球计数板在苂光显微镜 (OLYMPUS IX73) 明场下对藻细胞进行计数 (上下计数板总细胞数误差< $5 \%$ ), 对照组藻细胞数为 $Y_{1}$, 提取胶被后藻细胞数为 $Y_{2}$. 
1.4.2 单糖组成分析 将获得的 RPS 、粘液、英膜/鞘样品溶液在磁力搅拌器上于 $60^{\circ} \mathrm{C}$ 蒸发至三分之一体积, 以达到浓缩效果 ${ }^{[24]}$, 采用高效液相色谱法 (HPLC) 测定单糖组成 (中科百测科技有限公司, 北京).

待测样品水解: 精确吸取 $250 \mu \mathrm{L}$ 样品水解液到 $5 \mathrm{~mL}$ EP 管中, 加人 $250 \mu \mathrm{L} 0.6 \mathrm{~mol} / \mathrm{L} \mathrm{NaOH} 、 500 \mu \mathrm{L} 0.4$ $\mathrm{mol} / \mathrm{L} \mathrm{PMP}$-甲醇, $70^{\circ} \mathrm{C}$ 反应 $1 \mathrm{~h}$. 冷水中冷却 $10 \mathrm{~min}$; 加人 $500 \mu \mathrm{L} 0.3 \mathrm{~mol} / \mathrm{L} \mathrm{HCl}$ 中和, 再加人 $1 \mathrm{~mL}$ 氯仿漩浴 $1 \mathrm{~min}, 3000 \mathrm{r} / \mathrm{min}$ 离心 $10 \mathrm{~min}$, 小心取上清, 萃取 3 次后取上清液.

对照溶液: 精密称取甘露糖( M103970, 阿拉丁)、核糖(B21897, 源叶)、鼠李糖(B21172, 源叶)、葡萄糖 醛酸 (B25302, 源叶)、半乳糖醛酸 (B21894, 源叶)、氨基葡萄糖 ( SG8490, 索莱宝)、葡萄糖 (110833, 奥 科)、氨基半乳糖 (SG8450, 索莱宝)、半乳糖 (SG8010, 索莱宝)、岩藻糖 (B25632, 源叶)、木糖 (A600998, 生

工)、阿拉伯糖(B25845, 源叶) 对照品适量, 溶解稀释至各糖浓度为 $50 \mu \mathrm{g} / \mathrm{mL}$ 的混合对照溶液.

样品衍生: 分别精确吸取 $250 \mu \mathrm{L}$ 混合对照溶液和样品水解液到 $5 \mathrm{~mL} \mathrm{EP}$ 管中, 加人 $250 \mu \mathrm{L} 0.6 \mathrm{~mol} / \mathrm{L}$ $\mathrm{NaOH}, 500 \mu \mathrm{L} 0.4 \mathrm{~mol} / \mathrm{L}$ PMP-甲醇, $70^{\circ} \mathrm{C}$ 反应 $1 \mathrm{~h}$. 冷水中冷却 $10 \mathrm{~min}$; 加人 $500 \mu \mathrm{L} 0.3 \mathrm{~mol} / \mathrm{L} \mathrm{HCl}$ 中和, 再加 人 $1 \mathrm{~mL}$ 氯仿漩浴 $1 \mathrm{~min}, 3000 \mathrm{r} / \mathrm{min}$ 离心 $10 \mathrm{~min}$, 小心取上清, 萃取 3 次. 上清用于 HPLC.

色谱分析条件: 色谱柱: Xtimate C18 柱, $4.6 \mathrm{~mm} \times 200 \mathrm{~mm}$; 流动相: $0.05 \mathrm{~mol} / \mathrm{L} \mathrm{KH}_{2} \mathrm{PO}_{4}$ (氢氧化钠调节 $\mathrm{pH}=6.70)$-乙腈 $=83-17$; 柱温 $: 30^{\circ} \mathrm{C}$; 进样量: $20 \mu \mathrm{L}$; 流速: $1.0 \mathrm{~mL} / \mathrm{min}$; 检测波长: $250 \mathrm{~nm}$.

\section{5 数据处理}

所有实验重复 3 次, 数据用平均值 \pm 标准偏差表示. 数据分析采用 Origin Version 9.0( Origin Lab Corporation, USA) 方差分析和组间差异显著性检验 (One-way ANOVA), 当 $P<0.05$ 时, 处理组与对照组间存在显著 性差异. 图片数据亦为实验重复 3 次后的结果.

\section{2 结果}

\section{1 不同提取方法对蓝藻胶被的提取效果}

不同提取方法对蓝藻胶被的提取效果见表 4. 离心法和热水水浴法对蓝藻胶被提取效果不显著. 超声法 和阳离子交换树脂法对胶被提取有部分效果. 一定浓度、温度的硫酸水浴法和氢氧化钠水浴法对鱼腥藻胶 被提取效果明显; 氢氧化钠水浴法可以使念珠藻鞘结构变得松散, 而后通过超声可将藻丝与胶被分离.

表 4 不同提取方法对蓝藻胶被的提取效果

Tab.4 Effects of different extraction methods on the cyanobacterial surface coat

\begin{tabular}{|c|c|c|c|c|c|c|c|c|}
\hline 藻种 & $\begin{array}{l}\text { 胶被 } \\
\text { 类型 }\end{array}$ & 离心法 & 超声法 & $\begin{array}{c}\text { 阳离子交换 } \\
\text { 树脂法 }\end{array}$ & $\begin{array}{c}\text { 热水 } \\
\text { 提取法 }\end{array}$ & $\begin{array}{l}\text { 硫酸 } \\
\text { 水浴法 }\end{array}$ & $\begin{array}{c}\text { 氢氧化钠 } \\
\text { 水浴法 }\end{array}$ & $\begin{array}{c}\text { 适宜提取 } \\
\text { 方法 }\end{array}$ \\
\hline $\begin{array}{c}\text { FACHB-82 } \\
\text { 鱼腥藻 }\end{array}$ & $\begin{array}{l}\text { 荚膜+ } \\
\text { 粘液 }\end{array}$ & $x$ & $\begin{array}{l}\text { * 打散成短单链, } \\
\text { 但链周围荚膜依 } \\
\text { 然存在, 见图 2a }\end{array}$ & $\begin{array}{l}* \text { 会使藻链解聚成短 } \\
\text { 链, 但链周围英膜依 } \\
\text { 然明显存在, 见图 } 2 b\end{array}$ & 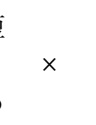 & V & V & $\begin{array}{l}\text { 硫酸水浴法/ } \\
\text { 氢氧化钠水 } \\
\text { 浴法, 见图 } 2 c\end{array}$ \\
\hline $\begin{array}{c}\text { FACHB-599 } \\
\text { 念珠藻 }\end{array}$ & 鞘 & $\begin{array}{c}* \text { 离心 } 15000 \mathrm{~g}, \\
1 \mathrm{~h} \text { 以上鞘结构裂 } \\
\text { 开, 小部分藻链逸 } \\
\text { 出, 见图 } 2 \mathrm{~d}\end{array}$ & $\begin{array}{c}* \text { 容易导致细胞 } \\
\text { 破裂, 见图 } 2 \mathrm{e}\end{array}$ & $\begin{array}{c}* \text { 能提取最外层鞘, } \\
\text { 大多鞘依然存在, } \\
\text { 见图 } 2 \mathrm{f}\end{array}$ & $x$ & $x$ & $\begin{array}{l}* \text { 可以使鞘结 } \\
\text { 构变得松散, } \\
\text { 见图 } 2 \mathrm{~g} \text { 、图 } 2 \mathrm{~h}\end{array}$ & $\begin{array}{l}\text { 氢氧化钠水 } \\
\text { 浴法+超声 } \\
\text { 法, 见图 } 2 \mathrm{i}\end{array}$ \\
\hline
\end{tabular}

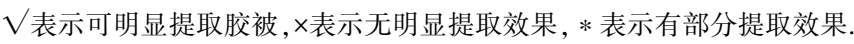

图 2a 显示鱼腥藻藻丝通过超声法均可打散成短单链,但藻丝周围荚膜依然存在. 通过阳离子交换树脂 法, 藻丝解聚成短丝, 但周围荚膜依然存在 (图 $2 \mathrm{~b}$ ). 硫酸水浴法 $\left(1 \mathrm{~mol} / \mathrm{L} \mathrm{H}_{2} \mathrm{SO}_{4}\right.$, 分别为 $50^{\circ} \mathrm{C}, 40 \mathrm{~min} 、 60^{\circ} \mathrm{C}$, $\left.30 \mathrm{~min} 、 70^{\circ} \mathrm{C}, 20 \mathrm{~min}\right)$ 和氢氧化钠水浴法 $\left(0.01 \mathrm{~mol} / \mathrm{L} \mathrm{NaOH}\right.$, 分别为 $\left.50^{\circ} \mathrm{C}, 20 \mathrm{~min} 、 60^{\circ} \mathrm{C}, 40 \mathrm{~min} 、 70^{\circ} \mathrm{C}, 30 \mathrm{~min}\right)$ 均可将荚膜提取完全 (图 2c).

念珠藻在高速离心 $(>15000 \mathrm{~g},>1 \mathrm{~h})$ 后, 鞘结构发生小部分破裂, 少部分藻链逸出, 经印度墨水染色发现 逸出藻链周围无胶被存在 (图 2d). 超声法对胶被提取效果不明显, 且功率过高容易导致细胞破裂 (图 $2 \mathrm{e}$ ). 阳离子交换树脂法只能提取最外层鞘, 内层鞘提取效果不明显(图 2f). 在温度、时间阈值内适当提高温度、 
延长处理时间,氢氧化钠水浴法 $\left(0.01 \mathrm{~mol} / \mathrm{L}, 80^{\circ} \mathrm{C}, 40 \mathrm{~min}\right)$ 可以使鞘结构变得松散 (图 $\left.2 \mathrm{~g}\right)$, 但胶被依然存在 ( 图 2h), 再用超声法冰浴超声 ( $20 \mathrm{kHz}, \geqslant 30 \mathrm{~W}, \geqslant 2 \mathrm{~min}$, on: $3 \mathrm{~s}$, off : $3 \mathrm{~s}$ ), 即可将念珠藻藻丝与鞘层完全 分离(图 $2 \mathrm{i}$ ).
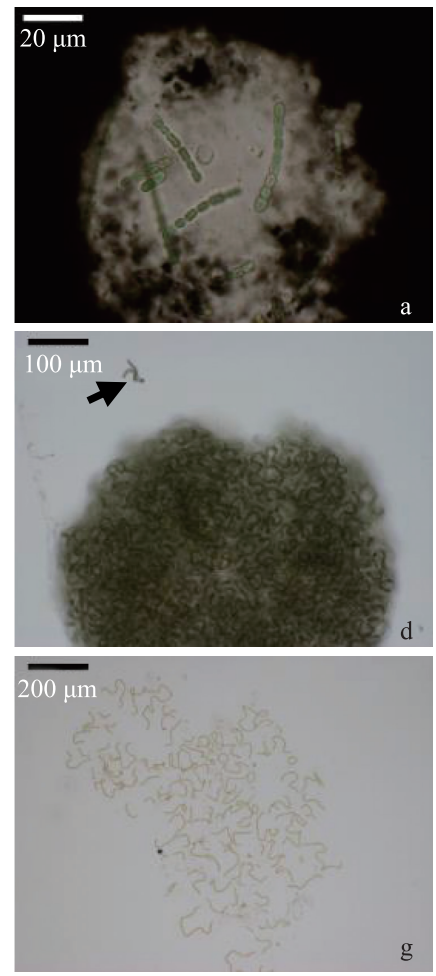
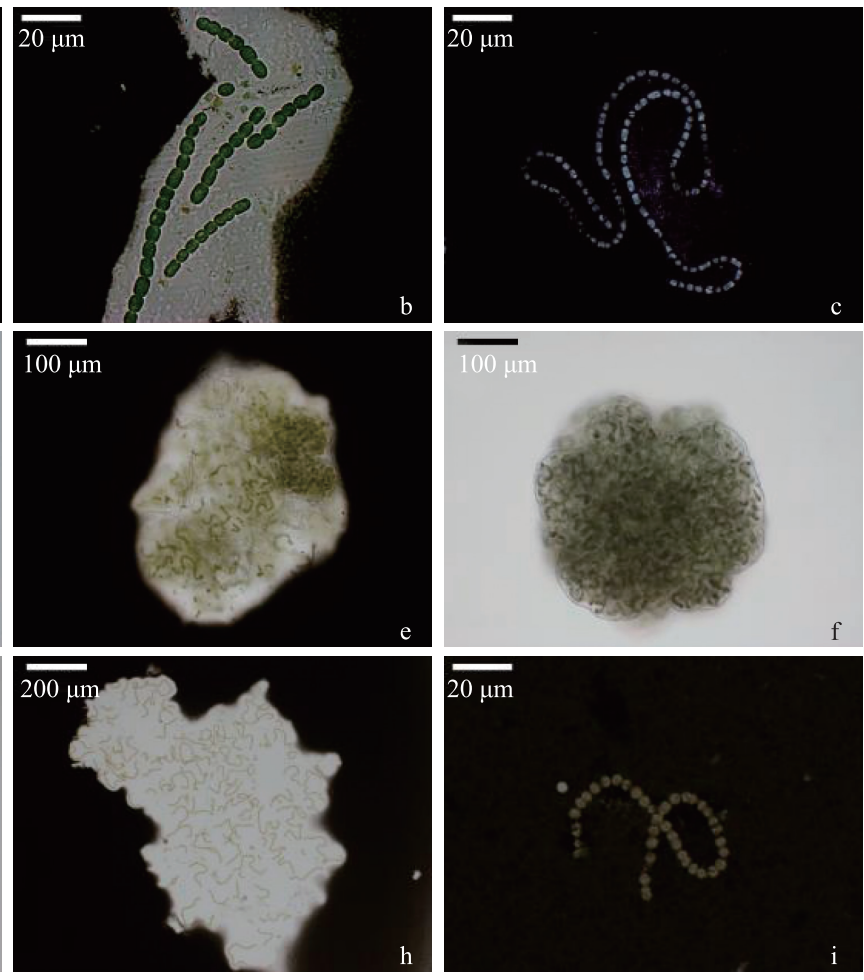

图 2 不同提取方法对蓝藻胶被形态的影响: ( a c ) FACHB-82 鱼腥藻; ( d i ) FACHB-599 念珠藻 （a:超声法处理,印度墨水染色; b : 阳离子交换树脂法处理,印度墨水染色; : 硫酸水浴法/

氢氧化钠水浴法处理,印度墨水染色; d: 离心法处理,逸出藻链 (箭头) ; e : 超声法处理, 印度墨水染色; $\mathrm{f}$ : 阳离子交换树脂法处理; $\mathrm{g}$ : 氢氧化钠水浴法处理; $\mathrm{h}$ : 氢氧化钠水浴法处理, 印度墨水染色; i : 氢氧化钠水浴法+超声法处理,印度墨水染色)

Fig. 2 Effects of different extraction methods on the morphology of cyanobacterial surface coat:

(a-c) FACHB-82 Anabaena sp.; (d-i) FACHB-599 Nostoc sp.

( a: ultrasonic method, stained with India ink; b: cation exchange resin method, stained with India ink; c: sulfuric acid/sodium hydroxide bath, stained with India ink;d: centrifugation, escaped algal filament ( arrow); e: ultrasonic method, stained with India ink;

$\mathrm{f}$ : cation exchange resin method;g: sodium hydroxide bath; $\mathrm{h}$ : sodium hydroxide bath, stained with India ink; i: sodium hydroxide bath and ultrasonic method, stained with India ink)

\section{2 蓝藻胶被提取方法条件优化}

鱼腥藻胶被完全提取组的藻细胞存在部分自发荧光, 菼光均较弱 (图 3), 而仅通过自发菼光的观察无法 选取鱼腥藻胶被的最优提取方法. 鱼腥藻胶被完全提取组和对照组的藻细胞数如图 4 所示, 处理组 A1 (1 $\left.\mathrm{mol} / \mathrm{L} \mathrm{H}_{2} \mathrm{SO}_{4}, 50^{\circ} \mathrm{C}, 40 \mathrm{~min}\right) 、 \mathrm{~A} 3\left(1 \mathrm{~mol} / \mathrm{L} \mathrm{H}_{2} \mathrm{SO}_{4}, 70^{\circ} \mathrm{C}, 20 \mathrm{~min}\right) 、 \mathrm{~B} 2\left(0.01 \mathrm{~mol} / \mathrm{L} \mathrm{NaOH}, 60^{\circ} \mathrm{C}, 40 \mathrm{~min}\right) 、 \mathrm{~B} 3$ $\left(0.01 \mathrm{~mol} / \mathrm{L} \mathrm{NaOH}, 70^{\circ} \mathrm{C}, 30 \mathrm{~min}\right)$ 与对照组相比, 细胞密度均出现显著性降低 $(P<0.05)$, 其中 $\mathrm{A} 3 、 \mathrm{~B} 2$ 组为极 显著差异 $(P<0.01)$. 处理组 $\mathrm{A} 2\left(1 \mathrm{~mol} / \mathrm{L} \mathrm{H}_{2} \mathrm{SO}_{4}, 60^{\circ} \mathrm{C}, 30 \mathrm{~min}\right) 、 \mathrm{~B} 1\left(0.01 \mathrm{~mol} / \mathrm{L} \mathrm{NaOH}, 50^{\circ} \mathrm{C}, 20 \mathrm{~min}\right)$ 细胞密 度分别达到 $(501.67 \pm 12.58) \times 10^{7}$ cells $/ \mathrm{L}$ 和 $(495.33 \pm 6.03) \times 10^{7}$ cells $/ \mathrm{L}$, 占对照组的 $98.8 \%$ 和 $97.6 \%$, 与对照 
无显著差异.

念珠藻在高温、长时间的氢氧化钠水浴法 $\left(0.01 \mathrm{~mol} / \mathrm{L}, 80^{\circ} \mathrm{C}, 40 \mathrm{~min}\right)$ 条件下鞘结构才变得松散,在光学 显微镜下未见明显边缘( 图 $2 \mathrm{~g}$ ), 由于超声功率过大, 时间过长易对藻细胞产生一定的机械破坏, 因此选择低 功率、短时间冰浴超声 ( $20 \mathrm{kHz}, 30 \mathrm{~W}, 2 \mathrm{~min}$, on: $3 \mathrm{~s}$, off: $3 \mathrm{~s}$ ) 结合处理即可将胶被和藻丝完全分离 (图 $2 \mathrm{i}$ ), 且自发菼光依然存在 (图 3C).
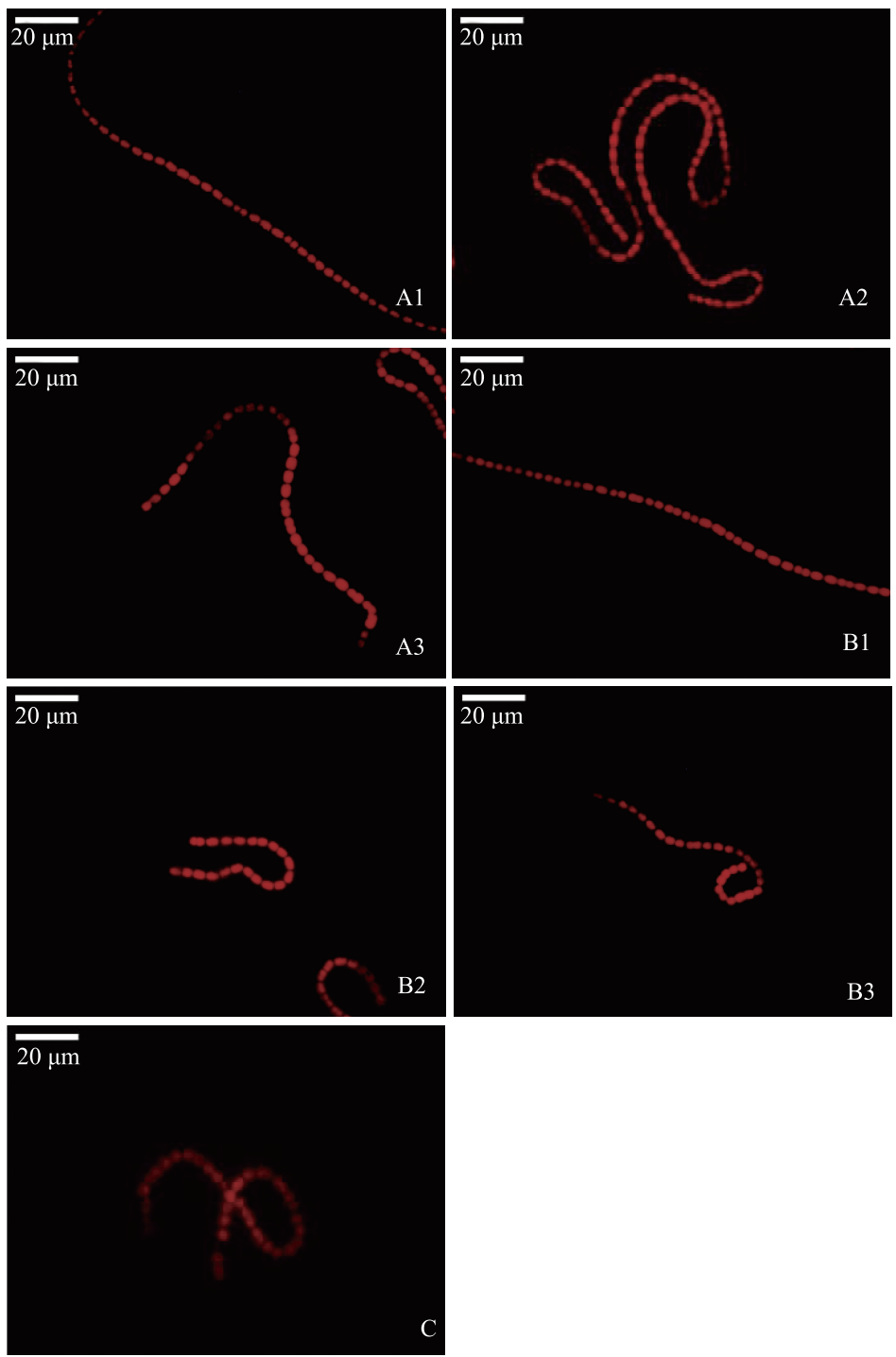

图 3 蓝藻完全提取胶被处理组自发苂光: (A、B) FACHB-82 鱼腥藻; ( C) FACHB-599 念珠藻

( $\mathrm{A} 1$ ( $\left.1 \mathrm{~mol} / \mathrm{L} \mathrm{H}_{2} \mathrm{SO}_{4}, 50^{\circ} \mathrm{C}, 40 \mathrm{~min}\right)$; $\mathrm{A} 2\left(1 \mathrm{~mol} / \mathrm{L} \mathrm{H}_{2} \mathrm{SO}_{4}, 60^{\circ} \mathrm{C}, 30 \mathrm{~min}\right)$; $\mathrm{A} 3\left(1 \mathrm{~mol} / \mathrm{L} \mathrm{H}_{2} \mathrm{SO}_{4}, 70^{\circ} \mathrm{C}, 20 \mathrm{~min}\right)$; $\mathrm{B} 1\left(0.01 \mathrm{~mol} / \mathrm{L} \mathrm{NaOH}, 50^{\circ} \mathrm{C}, 20 \mathrm{~min}\right)$; B2 ( $\left.0.01 \mathrm{~mol} / \mathrm{L} \mathrm{NaOH}, 60^{\circ} \mathrm{C}, 40 \mathrm{~min}\right)$; $\mathrm{B} 3\left(0.01 \mathrm{~mol} / \mathrm{L} \mathrm{NaOH}, 70^{\circ} \mathrm{C}, 30 \mathrm{~min}\right)$; $\mathrm{C}\left(0.01 \mathrm{~mol} / \mathrm{L} \mathrm{NaOH}, 80^{\circ} \mathrm{C}, 40 \mathrm{~min}+20 \mathrm{kHz}, 30 \mathrm{~W}, 2 \mathrm{~min}\right.$, on: $3 \mathrm{~s}$, off: $\left.\left.3 \mathrm{~s}\right)\right)$

Fig.3 Spontaneous fluorescence of cyanobacteria in the experimental treatment groups: ( A, B) FACHB-82 Anabaena sp. ; (C) FACHB-599 Nostoc sp. 


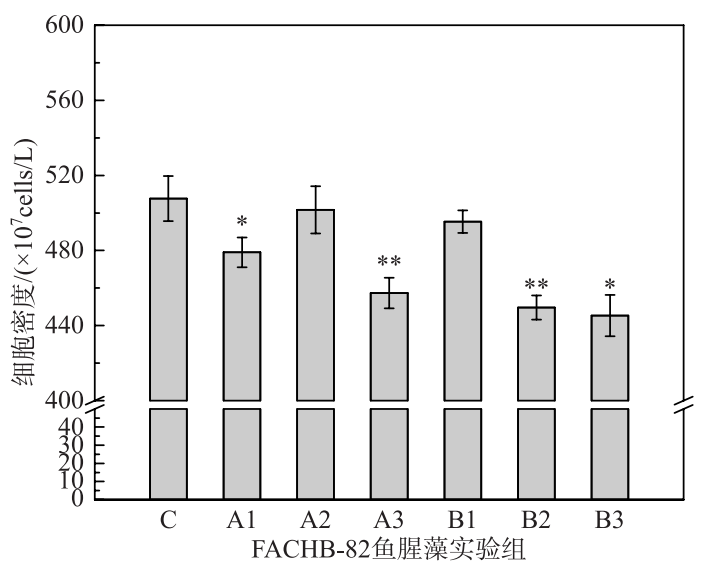

图 4 鱼腥藻完全提取胶被实验组下藻细胞密度

( $\mathrm{A} 1$ ( $1 \mathrm{~mol} / \mathrm{L} \mathrm{H}_{2} \mathrm{SO}_{4}, 50^{\circ} \mathrm{C}, 40 \mathrm{~min}$ ); $\mathrm{A} 2\left(1 \mathrm{~mol} / \mathrm{L} \mathrm{H}_{2} \mathrm{SO}_{4}, 60^{\circ} \mathrm{C}, 30 \mathrm{~min}\right)$; $\mathrm{A} 3\left(1 \mathrm{~mol} / \mathrm{L} \mathrm{H}_{2} \mathrm{SO}_{4}\right.$, $\left.70^{\circ} \mathrm{C}, 20 \mathrm{~min}\right) ; \mathrm{B} 1\left(0.01 \mathrm{~mol} / \mathrm{L} \mathrm{NaOH}, 50^{\circ} \mathrm{C}, 20 \mathrm{~min}\right) ; \mathrm{B} 2\left(0.01 \mathrm{~mol} / \mathrm{L} \mathrm{NaOH}, 60^{\circ} \mathrm{C}, 40 \mathrm{~min}\right)$; B3 ( $\left.0.01 \mathrm{~mol} / \mathrm{L} \mathrm{NaOH}, 70^{\circ} \mathrm{C}, 30 \mathrm{~min}\right) ; \mathrm{C}$ (未处理); $*, P<0.05 ; * *, P<0.01$ )

Fig.4 Cell density of Anabaena sp. in the experimental treatment groups

\section{3 不同类型胶被的单糖成分分析}

将上述对藻细胞苂光活性和细胞数无显著影响的方法用于鱼腥藻和念珠藻不同胶被类型的提取,鱼腥 藻采用氢氧化钠水浴法 $\left(0.01 \mathrm{~mol} / \mathrm{L} \mathrm{NaOH}, 50^{\circ} \mathrm{C}, 20 \mathrm{~min}\right)$, 念珠藻采用氢氧化钠水浴法 $\left(0.01 \mathrm{~mol} / \mathrm{L}, 80^{\circ} \mathrm{C}, 40\right.$ $\min$ )+超声法 ( $20 \mathrm{kHz}, 30 \mathrm{~W}, 2 \mathrm{~min}$, on: $3 \mathrm{~s}$, off: $3 \mathrm{~s}$ ) 进行胶被提取, 并对其进行多糖组分分析. 结果如表 5 所示, 鱼腥藻胶被包含粘液、荚膜, 且培养基中存在 RPS. RPS、粘液、荚膜三者的单糖总含量比约为 $2: 1: 15$, RPS 和粘液的单糖含量较少, 仅有 $5.581 \pm 0.24$ 和 $2.823 \pm 0.25 \mathrm{mg} / \mathrm{g}$, 荚膜单糖总含量为 $43.673 \pm 0.71 \mathrm{mg} / \mathrm{g}$. RPS 和粘液中占比最高的均为阿拉伯糖, 分别达到 $36.8 \%$ 和 $75.4 \%$, 而荚膜中最主要的单糖为葡萄糖, 占比 为 $32.5 \%$. 此外, 与 RPS 和粘液相比, 荚膜中还含有氨基葡萄糖 $(1.261 \pm 0.02 \mathrm{mg} / \mathrm{g})$.

念珠藻胶被只含鞘, 且未分泌 RPS 人培养基中. 鞘的总单糖含量达到 $111.719 \pm 4.51 \mathrm{mg} / \mathrm{g}$, 葡萄糖占比 最高, 为 $32.4 \%$, 此外, 念珠藻中含有鱼腥藻所没有的葡萄糖醛酸 $(5.066 \pm 0.24 \mathrm{mg} / \mathrm{g})$ 、鼠李糖 $(0.652 \pm 0.05$ $\mathrm{mg} / \mathrm{g})$ 和木糖 $(10.313 \pm 0.64 \mathrm{mg} / \mathrm{g})$.

\section{3 讨论}

蓝藻胶被主要以 3 种形态 (鞘、荚膜和粘液) 存在,这些胶被形态有时在藻细胞中是并存的. 胶被作为藻 细胞与外界环境间的缓冲基质, 可抵御外界不良环境以及参与营养物质的传输, 且不同类型胶被发挥的生 物学功能不同 ${ }^{[23,32-35]}$. 本研究在不显著影响藻类细胞活性和细胞完整性的前提下完全提取不同形态胶被, 为后续分析胶被生物学功能提供技术支撑. 对已有文献进行归纳 (表 1), 发现影响胶被提取效果的主要因 素有温度、提取剂浓度、时间等. 通过表 1 确定各提取方法的因素阈值, 研究设置温度梯度 $50 、 60 、 70$ 和 $80^{\circ} \mathrm{C}$, 提取剂浓度梯度 $0.01 、 0.1$ 和 $1 \mathrm{~mol} / \mathrm{L}$, 时间梯度 $20 、 30$ 和 $40 \mathrm{~min}$, 离心速度 $15000 、 20000 、 25000$ 和 $30000 \mathrm{~g}$, 超声功率 $30 、 60 、 90$ 和 $120 \mathrm{~W}$ 等, 设计对应双因素和正交实验, 针对不同形态的胶被区别提取, 通过 印度墨水染色和藻细胞自发苂光观察、细胞密度测定, 确定蓝藻不同形态胶被最优提取方法.

胶被是蓝藻细胞外的多层保护结构, 因此, 温和或机械方法无法将其完全提取 ${ }^{[36]}$. 由于 RPS 溶解于培 养基中, 因此可通过过滤法直接获取蓝藻的 RPS. 粘液松散地分散在细胞周围, 采用离心法 $(10000 \mathrm{~g}, 10$ $\min$ ) 可将藻细胞和粘液分离 ${ }^{[16]}$. 本研究中, 鱼腥藻荚膜与细胞粘结十分紧密, 念珠藻鞘层结构致密且坚硬, 通过高速离心 $(>15000 \mathrm{~g})$ 和冰浴超声均无法将荚膜和鞘与藻细胞分离, 且超声功率过大、时间过长容易使藻 细胞破裂. 阳离子交换树脂中的 $\mathrm{Na}^{+}$虽然可与 $\mathrm{Ca}^{2+} 、 \mathrm{Mg}^{2+}$ 之间发生离子交换作用, 破坏 $\mathrm{Ca}^{2+}$ 和 $\mathrm{Mg}^{2+}$ 与胶被之 


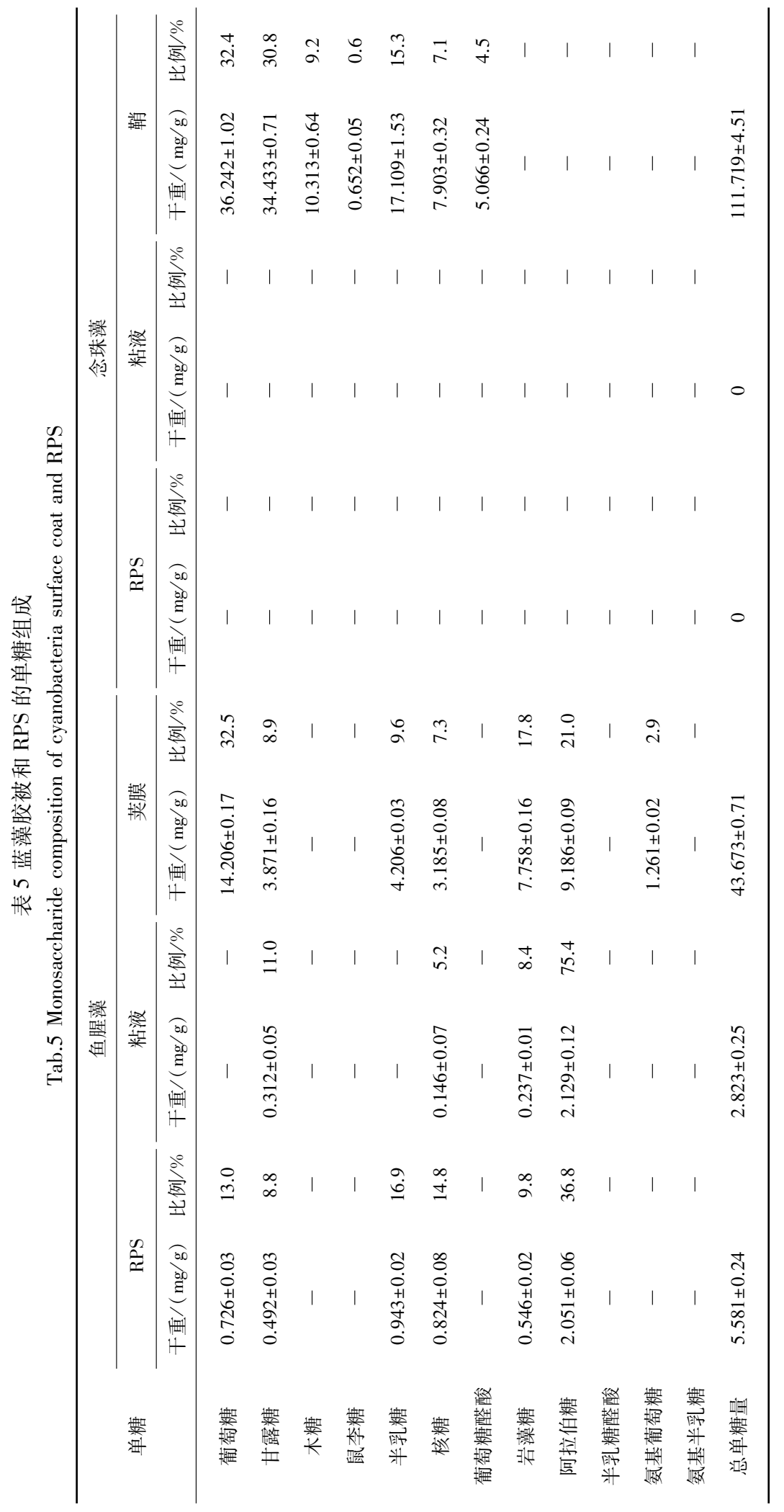


间的稳定结构, 但仅促使鱼腥藻藻丝解聚, 念珠藻失去最外层鞘, 未能完全提取荚膜和鞘, 这可能是由于阳 离子交换树脂的交换容量较小,不足以提取大量胶被.

研究结果显示, 使用提取剂的热水浴法对胶被的提取效果较好. 英膜可通过硫酸水浴法和氢氧化钠水 浴法完全提取.一方面, 温度升高使群体结构变得松散, 分子运动加剧, 增加胶被的可溶性 ${ }^{[36]}$; 另一方面, 硫 酸使溶液 $\mathrm{pH}$ 值降低, 引起颗粒之间表面电荷的变化, 导致群体藻细胞解散并改变胶被在水中的溶解度 ${ }^{[29]}$, 氢氧化钠可使胶被中的酸性基团分离, 在此作用下带负电荷的胶被相互排斥, 增加其溶解性. 本研究为减少 细胞裂解, 设置提取时间均小于 $1 \mathrm{~h}$, 发现 $1 \mathrm{~mol} / \mathrm{L} \mathrm{H}_{2} \mathrm{SO}_{4}$ 分别处理 $50^{\circ} \mathrm{C}, 40 \mathrm{~min} 、 60^{\circ} \mathrm{C}, 30 \mathrm{~min} 、 70^{\circ} \mathrm{C}, 20 \mathrm{~min}$ 以及 $0.01 \mathrm{~mol} / \mathrm{L} \mathrm{NaOH}$ 分别处理 $50^{\circ} \mathrm{C}, 20 \mathrm{~min} 、 60^{\circ} \mathrm{C}, 40 \mathrm{~min} 、 70^{\circ} \mathrm{C}, 30 \mathrm{~min}$ 均可完全提取荚膜. 此外, 鞘层通过 $0.01 \mathrm{~mol} / \mathrm{L} \mathrm{NaOH}$ 水浴法进一步高温、延长处理时间 $\left(80^{\circ} \mathrm{C}, 40 \mathrm{~min}\right.$ ), 结构才变得松散 (图 $2 \mathrm{~g}$ ). $\mathrm{Su}$ 等和 $\mathrm{Ge}$ 等 曾采用蒸馏水 $80^{\circ} \mathrm{C}$ 水浴 $6 \mathrm{~h}$ 提取念珠藻胶被 ${ }^{[26-27]}$, 推测与蒸馏水处理相比 $\mathrm{NaOH}$ 可以加速鞘层的溶解, 因此 其处理时间相对热水水浴法较短.

比较鱼腥藻完全提取荚膜方法下藻细胞的自发苂光, 发现各处理组自发菼光无明显差异 (图 3). 进一 步对各组提取后的藻细胞密度进行测定, 结果显示 $1 \mathrm{~mol} / \mathrm{L} 、 60^{\circ} \mathrm{C} 、 30 \mathrm{~min}$ 硫酸水浴法和 $0.01 \mathrm{~mol} / \mathrm{L} 、 50^{\circ} \mathrm{C} 、 20$ $\min$ 氢氧化钠水浴法提取后的藻细胞数无显著变化 $(P>0.05)$, 表明此条件适用于在不破坏细胞的完整性前 提下完全提取荚膜, 而其他处理组中的藻细胞均有不同程度的裂解. 有研究报道, 硫酸水浴法中酸的浓度要 求较高, 需达到 $1 \mathrm{~mol} / \mathrm{L}$, 才有利于胶被多糖的水解 ${ }^{[29]}$, 而在氢氧化钠水浴法中, 浓度较高不利于细胞的存 活 ${ }^{[36]}$, 因此 $\mathrm{NaOH}$ 浓度为 $0.01 \mathrm{~mol} / \mathrm{L}$ 为宜. 此外, 由于鞘层结构较英膜更坚硬 ${ }^{[15]}$, 其提取温度相较英膜更为 剧烈, $0.01 \mathrm{~mol} / \mathrm{L} 、 80^{\circ} \mathrm{C} 、 40 \mathrm{~min}$ 氢氧化钠水浴法处理, 念珠藻鞘层结构才变得松散. 为减少藻细胞破坏, 进一 步通过低功率、短时间冰浴超声 ( $20 \mathrm{kHz}, 30 \mathrm{~W}$, on: $3 \mathrm{~s}$, off: $3 \mathrm{~s}$ ) $2 \mathrm{~min}$ 后, 藻丝即与胶被完全分离. 用印度墨 水染色单根藻丝后观察显示其周围均无胶被结构, 由此推测鞘层结构虽致密且坚硬, 但与藻细胞之间的粘 附力不强.

大多数蓝藻胶被包含大量中性糖 (2 10 种) 与蛋白质分子结合 ${ }^{[37-38]}$. 将本研究中以最优方法提取出的 胶被及 RPS 进行了单糖组分分析, 鱼腥藻胶被中含有 7 种单糖组分, RPS 中含有 6 种, 念珠藻胶被中含有 7 种单糖组分. 鱼腥藻胶被中包含粘液 $(2.823 \pm 0.25 \mathrm{mg} / \mathrm{g}$ ) 和荚膜 ( $43.673 \pm 0.71 \mathrm{mg} / \mathrm{g}$ ), 并释放 RPS $(5.581 \pm$ $0.24 \mathrm{mg} / \mathrm{g})$ 到培养液中; 念珠藻胶被仅含鞘层 $(111.719 \pm 4.51 \mathrm{mg} / \mathrm{g})$, 不分泌 RPS. 有研究报道 RPS 是由荚膜 或粘液分泌的 ${ }^{[15]}$, 这和本研究在光学显微镜下观察的胶被类型及组分分析结果一致, 说明胶被大部分与藻 细胞结合并参与藻细胞生命活动, 少部分分泌到外界环境或松散地结合在藻细胞周围. 本研究中念珠藻均 为单个小群体外包裹鞘层结构, 群体之间没有粘附作用, 鱼腥藻则是藻丝粘附成大型群体生存. 这可能是因 为念珠藻鞘中含有脱氧糖 $(0.652 \pm 0.05 \mathrm{mg} / \mathrm{g}$ 鼠李糖 $)$, 从而使胶被产生疏水性 ${ }^{[6]}$, 未水化为粘液或 RPS. 另 一方面, 鱼腥藻荚膜中存在的氨基葡萄糖 $(1.261 \pm 0.02 \mathrm{mg} / \mathrm{g})$ 是念珠藻鞘层结构中没有的, 这些取代基可使 得荚膜比鞘更具有粘附性和凝结特性 ${ }^{[39]}$, 导致细胞与胶被间的联结十分牢固. 由此说明, 胶被单糖组分的 差异可能是造成荚膜和鞘的形态不同的原因之一. 此外, 念珠藻鞘中的葡萄糖醛酸 $(5.066 \pm 0.24 \mathrm{mg} / \mathrm{g})$, 使其 鞘可用碱性水溶液提取, 而鱼腥藻英膜中的氨基葡萄糖 $(1.261 \pm 0.02 \mathrm{mg} / \mathrm{g})$, 使其英膜可用酸性水溶液提 取 ${ }^{[00]}$. 本研究得到的对荚膜和鞘的最优提取方法也恰恰证实了这一点, 鱼腥藻英膜粘附性强, 在高速离心 作用下无法将藻细胞与荚膜分离, 而在 $50 \sim 60^{\circ} \mathrm{C}$ 氢氧化钠/硫酸水浴 $30 \mathrm{~min}$ 条件下可完全提取, 念珠藻的鞘 与藻细胞粘附力较弱但坚硬, 需更剧烈的方法 $\left(0.01 \mathrm{~mol} / \mathrm{L} \mathrm{NaOH}, 80^{\circ} \mathrm{C}, 40 \mathrm{~min}\right)$ 和冰浴超声 $(20 \mathrm{kHz}, 30 \mathrm{~W}$, $2 \mathrm{~min}$, on: $3 \mathrm{~s}$, off: $3 \mathrm{~s}$ ) 结合处理.

将本研究得到的荚膜和鞘的最优提取法分别运用到胶被形态以荚膜为主的纤细席藻 ( Phormidium tenue)、鞘丝藻 (Lyngbya sp.) 、微鞘藻 (Microcoleus sp.) 等丝状藻和胶被形态以鞘为主的卵囊藻 (Oocystis sp.)、雨生红球藻 (Haematococcus pluvialis) 等藻种, 均有很好的提取效果, 且提取后的藻细胞结构完整并具有 一定的活性(数据未显示). 在今后的研究中, 可针对有胶被和脱胶被的藻种进行营养盐吸收、抗胁迫能力等 过程的对比分析,进一步明确藻类胶被的生物学功能.

\section{4 参考文献}

[ 1 ] Bennett HS. Morphological aspects of extracellular polysaccharides. Journal of Histochemistry \& Cytochemistry, 1963,11 
(1) : 14-23.

[ 2 ] Qu F, Liang H, He J et al. Characterization of dissolved extracellular organic matter ( dEOM) and bound extracellular organic matter (bEOM) of Microcystis aeruginosa and their impacts on UF membrane fouling. Water Research, 2012,46 (9) : 2881-90.

[ 3 ] Chentir I, Hamdi M, Doumandji A et al. Enhancement of extracellular polymeric substances ( EPS) production in Spiruli$n a$ (Arthrospira sp.) by two-step cultivation process and partial characterization of their polysaccharidic moiety. International Journal of Biological Macromolecules, 2017, 105(2): 1412-1420.

[ 4 ] Pannard A, Pédrono J, Bormans M et al. Production of exopolymers (EPS) by cyanobacteria: impact on the carbon-to-nutrient ratio of the particulate organic matter. Aquatic Ecology, 2015, 50(1) : 29-44.

[ 5 ] Hou J, Yang Y, Wang $\mathrm{P}$ et al. Effects of $\mathrm{CeO}_{2}, \mathrm{CuO}$, and $\mathrm{ZnO}$ nanoparticles on physiological features of Microcystis aeruginosa and the production and composition of extracellular polymeric substances. Environmental Science and Pollution Research International, 2017, 24(1) : 226-235.

[ 6 ] Ahmed M, Moerdijk-Poortvliet TC, Wijnholds A et al. Isolation, characterization and localization of extracellular polymeric substances from the cyanobacterium Arthrospira platensis strain MMG-9. European Journal of Phycology, 2014, 49(2) : 143-150.

[ 7 ] Richert L, Golubic S, Guedes RL et al. Characterization of exopolysaccharides produced by cyanobacteria isolated from Polynesian microbial mats. Current Microbiology, 2005, 51(6) : 379-84.

[ 8 ] Ge H, Zhang J, Zhou X et al. Effects of light intensity on components and topographical structures of extracellular polymeric substances from Microcoleus vaginatus (Cyanophyceae). Phycologia, 2014, 53(2) : 167-173.

[ 9 ] Lange W. Speculations on a possible essential function of the gelatinous sheath of blue-green algae. Canadian Journal of Microbiology, 1976, 22(8) : 1181-1185.

[10] Reynolds CS. Variability in the provision and function of mucilage in phytoplankton: Facultative responses to the environment. Hydrobiologia, 2007, 578(1) : 37-45.

[11] Yoshimura H, Kotake T, Aohara T et al. The role of extracellular polysaccharides produced by the terrestrial cyanobacterium Nostoc sp. strain HK-01 in NaCl tolerance. Journal of Applied Phycology, 2012, 24(2) : 237-243.

[12] Mishra A, Tandon R, Kesarwani S et al. Emerging applications of cyanobacterial ultraviolet protecting compound scytonemin. Journal of Applied Phycology, 2015, 27(3) : 1045-1051.

[13] Weiss TL, Roth R, Goodson C et al. Colony organization in the green alga Botryococcus braunii ( Race B) is specified by a complex extracellular matrix. Eukaryotic Cell, 2012, 11(12): 1424-1440.

[14] Liu L, Huang Q, Qin B. Characteristics and roles of Microcystis extracellular polymeric substances ( EPS) in cyanobacterial blooms: a short review. Journal of Freshwater Ecology, 2018, 33(1) : 183-193.

[15] De Philippis R and Vincenzini M. Exocellular polysaccharides from cyanobacteria and their possible applications. FEMS Microbiology Reviews, 1998, 22(3): 151-175.

[16] Kuang X, Shao J, Chen A et al. Effects of bloom-forming cyanobacterial extracellular polymeric substances on the adsorption of cadmium onto kaolinite: Behaviors and possible mechanisms. Springerplus, 2016, 5: 542.

[17] Yang Y, Hou J, Wang P et al. The effects of extracellular polymeric substances on magnetic iron oxide nanoparticles stability and the removal of microcystin-LR in aqueous environments. Ecotoxicol Environmental Safety, 2018, 148: 89-96.

[18] Liu L, Huang Q, Zhang Y et al. Excitation-emission matrix fluorescence and parallel factor analyses of the effects of $\mathrm{N}$ and $\mathrm{P}$ nutrients on the extracellular polymeric substances of Microcystis aeruginosa. Limnologica, 2017, 63: 18-26.

[19] Shen G, Qu D, Li K et al. Composition of extracellular and intracellular polymeric substances produced by Scenedesmus and Microcystis. Environmental Engineering Science, 2017, 34(12) : 887-894.

[20] Li M, Zhu W, Sun Q. Solubilisation of mucilage induces changes in Microcystis colonial morphology. New Zealand Journal of Marine and Freshwater Research, 2014, 48(1): 38-47.

[21 Frølund B, Palmgren R, Keiding K et al. Extraction of extracellular polymers from activated sludge using a cation exchange resin. Water Research, 1996, 30(8) : 1749-1758.

[22] Fang HH, Jia X. Extraction of extracellular polymer from anaerobic sludges. Biotechnology Techniques, 1996, 10( 11): 803-808.

[23] Chen B, Li F, Liu N et al. Role of extracellular polymeric substances from Chlorella vulgaris in the removal of ammonium 
and orthophosphate under the stress of cadmium. Bioresource Technology, 2015, 190: 299-306.

[24] Mishra A, Jha B. Isolation and characterization of extracellular polymeric substances from micro-algae Dunaliellasalina under salt stress. Bioresource Technology, 2009, 100(13) : 3382-6.

[25] Xu H, Cai H, Yu G et al. Insights into extracellular polymeric substances of cyanobacterium Microcystis aeruginosa using fractionation procedure and parallel factor analysis. Water Research, 2013, 47(6) : 2005-14.

[26] Su J, Jia S, Chen X et al. Morphology, cell growth, and polysaccharide production of Nostoc flagelliforme in liquid suspension culture at different agitation rates. Journal of Applied Phycology, 2008, 20(3) : 213-217.

[27] Ge H, Xia L, Zhou X et al. Effects of light intensity on components and topographical structures of extracellular polysaccharides from the cyanobacteria Nostoc sp. Journal of Microbiology, 2014, 52(2) : 179-83.

[28] Liu L, Qin B, Zhang Y et al. Extraction and characterization of bound extracellular polymeric substances from cultured pure cyanobacterium (Microcystis wesenbergii). Journal of Environmental Sciences (China), 2014, 26( 8) : 1725-32.

[29] Eder M and Lutz-Meindl U. Analyses and localization of pectin-like carbohydrates in cell wall and mucilage of the green alga Netrium digitus. Protoplasma, 2010, 243(1-4) : 25-38.

[30] Takahashi E, Ledauphin J, Goux D et al. Optimising extraction of extracellular polymeric substances (EPS) from benthic diatoms: comparison of the efficiency of six EPS extraction methods. Marine and Freshwater Research, 2010, 60(12): 1201-1210.

[31] Pierre G, Zhao JM, Orvain F et al. Seasonal dynamics of extracellular polymeric substances (EPS) in surface sediments of a diatom-dominated intertidal mudflat (Marennes-Oléron, France). Journal of Sea Research, 2014, 92: 26-35.

[32] Fang L, Wei X, Cai P et al. Role of extracellular polymeric substances in Cu (II) adsorption on Bacillus subtilis and Pseudomonas putida. Bioresource Technology, 2011, 102(2) : 1137-1141.

[33] Volesky B, Holan Z. Biosorption of heavy metals. Biotechnology Progress, 1995, 11(3) : 235-250.

[34] Brookes JD, Ganf GG. Variations in the buoyancy response of Microcystis aeruginosa to nitrogen, phosphorus and light. Journal of Plankton Research, 2001, 23(12): 1399-1411.

[35] Yang Z, Kong F, Yang Z et al. Benefits and costs of the grazer-induced colony formation in Microcystis aeruginosa. Annales de Limnologie-International Journal of Limnology, 2009: 203-208. DOI: 10.1051/limn/2009020.

[36] Delattre C, Pierre G, Laroche C et al. Production, extraction and characterization of microalgal and cyanobacterial exopolysaccharides. Biotechnology Advances, 2016, 34(7) : 1159-1179.

[37] Otero A, Vincenzini M. Extracellular polysaccharide synthesis by Nostoc strains as affected by N source and light intensity. Journal of Biotechnology, 2003, 102(2) : 143-152.

[38] Mei QH, Miao YQ, Zhang CW et al. Preliminary studies on the isolation, purification and physicochemical properties of extracellular acidic polysaccharides from Microcystis aeruginosa var. major. J Lake Sci, 2005, 17(4) : 322-326. DOI: 10. 18307/2005.0407. [梅秋红, 缪月秋, 张成武等. 铜绿微囊藻 (Microcystic aeruginosa var. major) 胞外酸性多糖的分 离、纯化及其理化特性. 湖泊科学, 2005, 17(4) : 322-326.]

[39] Chen MZ, Xie MJ, Wu W et al. Chemical characteristics of capsular polysaccharide and water-soluble released exopolysaccharide from Microcystis. J Lake Sci, 2016, 28(3) : 609-615. DOI : 10.18307/2016.0317. [陈毛珍, 谢梅娟, 巫伟等. 微囊藻胶鞘多糖和水溶性胞外多糖的化学特性. 湖泊科学, 2016, 28(3): 609-615.]

[40] Pang N, Zhang JQ, Qi J et al. Research status of microbial exopolysaccharide and its metabolic pathway. Advances in Microbiology, 2017, 6(2) : 27-34. [ 庞宁, 张佳琪, 齐进等. 微生物胞外多糖及其生物合成途径研究现状. 微生物前 沿, 2017, 6(2): 27-34.] 